\title{
Research on Subdivision Algorithm of Linear Frequency Modulated Continuous Wave and MATLAB Simulation Analysis
}

\author{
Shu $\mathrm{Li}^{1, \mathrm{a}}$ and Yifan $\mathrm{Wu}^{2, \mathrm{~b}}$ \\ ${ }^{1}$ Beijing Institute of Petrol-Oil Research, Beijing, China, 102300 \\ ${ }^{2}$ Wuxi Leihua tech Co, Ltd., Wuxi, China, 214000 \\ afirefly_Is@163.com, ${ }^{b} 137473684 @ q q . c o m$
}

Keywords: Linear frequency modulated continuous wave; Frequency subdivision interpolation; MATLAB

\begin{abstract}
The principle of linear frequency modulated continuous wave and the characteristic of a special zigzag linear frequency modulated continuous wave are described in this paper, as well as ranging with linear frequency modulated continuous wave in radar application. Two algorithms of improving ranging accuracy are introduced, and iterative method and frequency subdivision interpolation method are discussed respectively through frequency subdivision estimation theory. Besides, MATLAB simulation analysis is utilized to compare the ranging reliability with the two methods, so as to point out the ranging accuracy of each method.
\end{abstract}

\section{Introduction}

The method of estimating signal frequency of linear frequency modulated continuous wave with FFT has been widely utilized in radar signal process, especially for its special characteristics in radar ranging. Continuous time-domain spectrum is changed into discrete frequency spectrum after FFT, for example, if the signal interval is $\mathrm{T}$, the delta frequency $\Delta f=1 / \mathrm{T}$. Therefore, the sinus signal frequency accuracy estimated directly with the frequency corresponding to the maximum FFT spectrum value is subject to the duration of monitoring time, error range of which is $\pm \Delta f / 2$. Increase of monitoring time can result in implementation difficulty because of too many FFT points, and moreover, it might not be possible to extend the monitoring time at will in some limited conditions. It is possible to utilize frequency subdivision method [1] to break through the frequency estimation accuracy limited by FFT frequency resolution, except that more calculation amount shall be added. When the signal frequency is not integral multiple of FFT frequency resolution, the Barrier Effect of FFT might result in frequency leakage, when the actual signal frequency locates between the two maximum spectrum lines inside FFT main lobe; therefore, it is possible to estimate the actual frequency position [8] between the two spectrum lines with the magnitude specific value of the second spectrum line over that of the maximum spectrum. In order to control side-lobe, window addition is usually adopted for sample data prior to FFT; the added window can widen the main lobe, so that more than three spectrums can appear inside the main lobe. Energy centrobaric method can improve frequency estimation accuracy with multiple spectrums inside main lobe. In case of no window added, it is easy to cause errors in determining the second spectrum when the actual signal frequency approaches the frequency corresponding to the maximum FFT spectrum, so that frequency interpolation direction errors might occur and limit the frequency interpolation accuracy with FFT magnitude specific value. The substitution of real part of specific value of the second maximum value over the maximum value inside FFT main lobe with magnitude specific value can eliminate the frequency estimation error caused by interpolation direction errors. The phase of FFT maximum value correlates to the deviation between actual signal frequency and the frequency corresponding to the maximum spectrum of FFT magnitude, but the primary phase of the signal is usually unknown, for which it is not possible to estimate the frequency with the phase of FFT maximum spectrum. It is possible to eliminate primary phase 
influences with segment FFT method, so as to improve the frequency estimation accuracy with FFT phase.

\section{Linear Frequency Modulated Continuous Wave}

A linear frequency modulated continuous wave radar can calculate the distance from target through changing emission signal frequency in time domain and measuring the frequency difference between received signals and transmitted signals. See the following diagram for the linear frequency modulated continuous wave radar system.

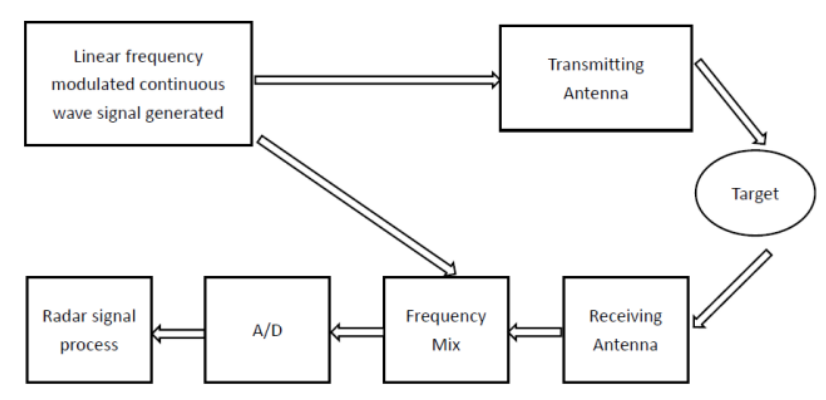

Figure 1. Linear frequency modulated continuous wave radar system

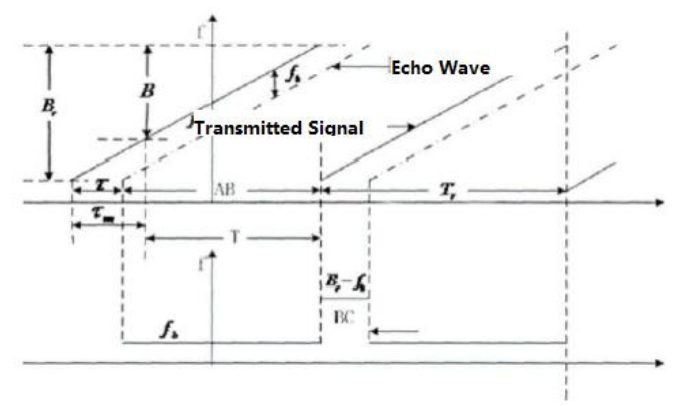

Figure 2. Transmitted signals and received signals

The generated linear frequency modulated continuous wave signals are transmitted via the transmitting antenna, before the rebounded signals from the target are received via the receiving antenna. The echo signals are mixed with some transmitted signals (the primary signals) before frequency difference signals are received; then, the target information is obtained through A/D conversion for frequency difference signals and signal process.

\section{Zigzag Linear Frequency Modulated Continuous Wave}

In linear frequency modulated continuous wave radar system, zigzag linear frequency modulated continuous wave is always utilized. The following diagram shows the relationship between the transmitted signals and received signals, where, $T_{r}$ means term; $\tau_{m}$ means target time delay; $\mathrm{B}_{\mathrm{r}}$ means bandwidth; $f_{0}$ means central frequency; frequency modulation slope $k=B_{r} / T_{r}$.

The upper part of the above diagram represents the frequency-time relationship of signal, where the solid line means transmitted signals; the dotted line means echo signals; there is a time delay of $\tau=2 R / c$ between the echo signals and transmitted signals. The lower part of the above diagram shows the frequency difference signal $f_{b}$, which is obtained through mixing of echo signals and transmitted primary signals. The valid segment of frequency difference is as follows

$$
f_{b}=\tau k=\frac{2 R B_{r}}{T_{r} C}
$$

The zigzag linear frequency modulated continuous wave can be represented as follows for its transmitted signals in the frequency modulation term

$$
S(t)=A \exp \left(j 2 \pi\left(f_{0}(t-n T)+k(t-t)^{2} / 2\right)\right)
$$

Where, A means the magnitude of transmitted signals; $f_{0}$ means the frequency of transmitted signals at $t=n T$. When $t=0$, the echo time delay is $\tau_{m}$, and the target echo signals can be represented as follows 


$$
S(t)=A \exp \left(j 2 \pi\left(f_{0}\left(t-n T-\tau_{m}\right)+k\left(t-t-\tau_{m}\right)^{2} / 2\right)\right)
$$

\section{Frequency Subdivision Research}

Actual signals are indefinitely long and their spectrum is continuous. However, for spectrum analysis via digital methods, the signals have to be cut short into definite length of $t$ with the method of adding square window. The signal term will be changed into spectrum after the window addition, and accordingly, the original continuous spectrum will be changed into discrete spectrum and the spectrum lines will only occur to the integral multiple position. Therefore, the actual signals between spectrum lines might be erased because of spectrum line obstacle, which is named as Barrier Effect. For example, if the cut signal length is $f_{0}=0.5 \mathrm{~s}$, the possible spectrum lines might be $2 \mathrm{~Hz}, 4 \mathrm{~Hz}, 6 \mathrm{~Hz}, 8 \mathrm{~Hz}, \ldots$; if a $7 \mathrm{~Hz}$ component is included in the signals, the component might be eliminated and not be shown. In this condition, it is possible to increase spectrum line density to improve inspection accuracy. However, the increase of sampling period might damage sampling principle and if sampling numbers is increased, the calculation amount might be considerably increased. Thus, the Barrier Effect might influence the frequency estimation algorithm accuracy based on FFT.

Iterative Method. Now, we investigate an echo signal of linear frequency pulse

$$
S(t)=\operatorname{rect}\left(\frac{t-\Delta t-n T_{p r i}}{T_{p}}\right) \exp \left(\mathrm{j} \pi \mathrm{k}(\mathrm{t}-\Delta \mathrm{t})^{2}\right)
$$

Where, $\Delta T$ means target time delay; $T_{p r i}$ means pulse term; $T_{p}$ means pulse width; $\mathrm{k}$ means frequency modulation slope; rect $(*)$ means square pulse block. Perform Fourier transformation, and you can get (induced with phase position POSP principle):

$$
S(t)=\operatorname{rect}\left(\frac{f}{k T_{p}}\right) \exp \left(-\mathrm{j} \pi \frac{f^{2}}{k}-j 2 \pi \Delta t f\right)
$$

We can form a reference function:

$$
R E F(f)=\exp \left(\mathrm{j} \pi \frac{f^{2}}{k}\right)
$$

Multiply it with $S(f)$ and add with Gauss white noise, and you can get:

$$
\begin{aligned}
& S_{1}(f)=\operatorname{rect}\left(\frac{f}{k T_{p}}\right) \exp (-j 2 \pi \Delta t f)+\mathrm{w}(\mathrm{t}) \\
& \mathrm{t}=0,1, \ldots, \mathrm{N}-1
\end{aligned}
$$

Where, $f$ and $\Delta T$ mean frequency and sampling interval; $w(t)$ means Gauss white noise series; $t$ means sample quantity. The maximum spectrum value is labeled with $\left|S\left(K_{0}\right)\right|$, and the frequency is estimated as follows:

$$
f=\left(k_{0}+\delta\right) \Delta f
$$

Where, $\Delta f=1 / N \Delta t$

Where, $\delta$ means remaining interval relating to $\mathrm{N}$, sample quantity of FFT. The frequency estimation purpose is to find accurate value of $\delta$. Since deviated from spectrum peak by FFT factor of 0.5 times of $\Delta f, p=+/-0.5$, for which the following formula can be achieved: 


$$
S_{k 0}(p)=h \frac{\delta}{\delta-p}
$$

Where $h=-N \exp (j \varphi)\left(1+e^{j 2 \pi \delta}\right) /(j 2 \pi \delta)$

The following formula can be obtained according to reference document No. 10:

$$
D\left(\delta_{0}\right)=0.5 \frac{b \delta /(0.5-\delta)-b \delta /(0.5+\delta)}{b \delta /(0.5-\delta)+b \delta /(0.5+\delta)}
$$

Eliminate noise, and you can get:

$$
D\left(\delta_{0}\right)=0.5 \frac{S_{k 0}(0.5)-S_{k 0} /(-0.5)}{S_{k 0}(0.5)+S_{k 0} /(-0.5)}
$$

According to the above arguments, the iterative process needs initialization first and FFT shall be done to Samples $\mathrm{N}$ for $S(t)$, and the result is labeled as $S(k)$ to find the position $k_{0}$ of maximum spectrum. Then, calculate $\delta_{i}=\delta_{i-1}+D\left(\delta_{i-1}\right)$, in which $i$ means iterative times.

Frequency Subdivision Interpolation Method. Similarly, perform FFT to Samples N for $S(t)$ to locate $k_{0}$ of maximum spectrum line. In consideration of symmetry of FFT, take only the first half of spectrum, e.g. N/2. The frequency resolution of FFT is $\Delta f=1 / \mathrm{T}$, and the observed spectrum is the interval between adjacent spectrums. Rough estimation result for the signal is $f=k_{0} \Delta f$. When signal frequency is not integral multiple of $\Delta f$, there are two spectrum lines corresponding to $S(k)$, and the magnitude of maximum spectrum is $S_{0}$. The secondary maximum spectrum magnitude is $S_{1}$; the position of secondary maximum spectrum is labeled as $k_{1}=k_{0} \pm 0.5$. The difference value between actual signal frequency and the roughly-estimated frequency is labeled as $\delta=\left(f_{0}-f\right) / \Delta f$. Define two boundary limit range through comparing the left and right secondary maximum spectrum values near the maximum value, and find the frequency $k_{m}$ of mximum magnitude values in sequence, so as to calculate the target distance as follows:

$$
R=\frac{\Delta t c}{2}=\frac{\Delta f c}{2 k}=\frac{\left(k_{m}-1\right) F_{s} c}{2 K N}
$$

Note: $K$ means frequency modulation slope.

\section{MATLAB Simulation Analysis}

MATLAB simulation analysis can be used to validate accuracy of the algorism discussed in this article. Compare the iterative method with the frequency subdivision interpolation, and the later one can achieve higher ranging accuracy after a certain cycles. Here's the summary of the process of the frequency subdivision interpolation:

1) $x-x_{a} \leq e$

When $x-x_{a}>x-x_{b}$, flag=2

When $x-x_{a} \leq x-x_{b}$, flag $=1$

2) $x-x_{b} \leq e$, flag $=2$

3) $x-x_{a}>e \& x-x_{b}>e$, flag $=0$

When $x_{a}<x_{b}, a=a+\Delta f$

When $x_{a}>x_{b}, b=a+\Delta f$ 
4) $x_{a}+e<x<x_{b}-e, a=a+\Delta f$,flag $=0$

5) $x_{b}+e<x<x_{a}-e, b=a+\Delta f$,flag $=0$

Using the developed MATLAB tools for analyzing the two ways of method we get the results diagram below Fig. 3 .

See the diagrams for the spectrum graph and the roughly-estimated ranging results. The position of maximum frequency magnitude is at 130 and the calculated ranging distance is $966.8 \mathrm{~cm}$, both of which cannot meet the accuracy requirements. Subdivide the maximum frequency value position with the iterative method and frequency subdivision interpolation method, and the target distance is calculated as the above table. It shows that the ranging accuracy of the frequency subdivision interpolation method is more stable after a certain recurrence cycles, so that $0.01 \mathrm{~mm}$ can be achieved.

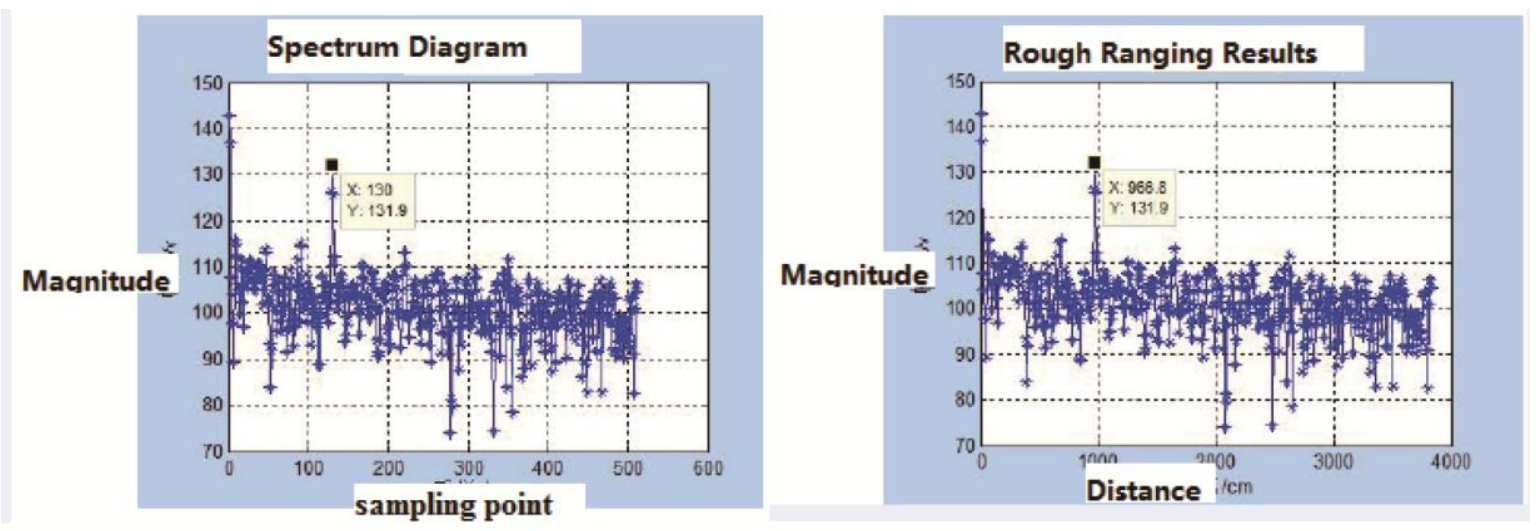

Figure 3. Spectrum diagram

Table 1 Target distance

\begin{tabular}{|c|c|c|c|}
\hline Iterative Times & rative Method $(\mathrm{cm})$ & currence Cycles & Frequency Subdivision Interpolation Method (cm) \\
\hline 1 & 966.6214 & 3 & 966.8307 \\
\hline 2 & 966.8307 & 6 & 966.7136 \\
\hline 3 & 966.7118 & 9 & 966.7209 \\
\hline 4 & 966.7119 & 12 & 966.7200 \\
\hline 5 & 966.7119 & 13 & 966.7204 \\
\hline 6 & 966.7119 & 14 & 966.7202 \\
\hline
\end{tabular}

\section{Conclusion}

To make a conclusion, two means of finding the target distance discussed in this paper have both accurate and efficient results in simulation. For more practical application, the frequency subdivision interpolation method provides a better a higher accuracy in measurement. Therefore, this algorithm is generally used in our industry.

\section{References}

[1] Li Yu, Liu Jingsen, Mechanism and Improvement of Direct Anonymous Attestation Scheme [J], Journal of Henan University, 2007, 37(2), P195-197 (Ch). 
[2] "Frequency-Modulated Continuous-Wave Radar". Radartutorial. Retrieved 2012-08-07.

[3] MENG Xiangping Gao Yan. Electric systems analysis [M]. Beijing: Higher Education Press, 2004.3-21.

[4] James M. Ridenour (1947). Radar System Engineering. MIT Radiation Lab series 1. p. 629.

[5] M. Ash et al., A New Multistatic FMCW Radar Architecture By Over-The-Air Deramping, IEEE Sensors Journal, No. 99, 2015.

[6] "Stalker Hand Held Police Laser". Stalker Radar. 